Pacific Journal of Mathematics

THE FIXED-POINT PARTITION LATTICES 


\section{THE FIXED-POINT PARTITION LATTICES}

\section{Phil HaNLON}

Let $\sigma$ be a permutation of the set $\{1,2, \cdots, n\}$ and let $\Pi(N)$ denote the lattice of partitions of $\{1,2, \cdots, n\}$. There is an obvious induced action of $\sigma$ on $\Pi(N)$; let $\Pi(N)_{\sigma}=L$ denote the lattice of partitions fixed by $\sigma$.

The structure of $L$ is analyzed with particular attention paid to $/ \mathscr{C}$, the meet sublattice of $L$ consisting of 1 together with all elements of $L$ which are meets of coatoms of $L$. It is shown that $\mathscr{C}$ is supersolvable, and that there exists a pregeometry on the set of atoms of $\mathscr{C}$ whose lattice of fiats $G$ is a meet sublattice of $\mathscr{C}$. It is shown that $G$ is supersolvable and results of Stanley are used to show that the Birkhofif polynomials $B,(\lambda)$ and $B_{G}(\lambda)$ are

$$
B_{G}(\lambda)=(\lambda-1)(\lambda-j) \cdots(\lambda-(m-1) j)
$$

and

$$
B .(\lambda)=(\lambda-1)^{r-1} B_{G}(\lambda) .
$$

Here $m$ is the number of cycles of $\sigma, j$ is square-free part of the greatest common divisor of the lengths of $\sigma$ and $r$ is the number of prime divisors of $j$. $\mathscr{C}$ coincides with $G$ exactly when $j$ is prime.

1. Preliminaries. Let $(P, \leqq)$ be a finite partially ordered set. An automorphism $\sigma$ of $(P, \leqq)$ is a permutation of $P$ satisfying $x \leqq y$ iff $x \sigma \leqq y \sigma$ for all $x, y \in P$. The group of all automorphisms of $P$ is denoted $\Gamma(P)$. For $\sigma \in \Gamma(P)$, let $P_{\sigma}=\{x \in P: x \sigma=x\}$. The set $P_{\sigma}$ together with the ordering inherited from $P$ is called the fixed point partial ordering of $\sigma$. If $P$ is lattice then $P_{\sigma}$ is a sublattice of $P$. To see this, let $x, y \in P_{\sigma}$. Then $(x \vee y) \sigma \geqq x \sigma=x$ and $(x \vee y) \sigma \geqq y \sigma=y$, so $(x \vee y) \sigma \geqq x \vee y$. If $(x \vee y) \sigma>x \vee y$, then $(x \vee y)<(x \vee y) \sigma<$ $(x \vee y) \sigma^{2}<\cdots$ forms an infinite ascending chain in $P$ which is impossible since $P$ is finite. So $(x \vee y) \sigma=x \vee y$ hence the set $P_{\sigma}$ is closed under joins in $P$. Similarly $P_{o}$ is closed under meets.

A partition $\rho$ of a finite set $\Omega=\left\{\omega_{1}, \cdots, \omega_{n}\right\}$ is a collection $\rho=B_{1} / B_{2} / \cdots / B_{k}$ of disjoint, nonempty subsets of $\Omega$ whose union is all of $\Omega$. The set of all partitions of $\Omega$ is denoted $\Pi(\Omega)$; if $\Omega=$ $\{1,2, \cdots, n\}$ this is written $\Pi(N) . \quad \Pi(\Omega)$ ordered by refinement is a lattice.

Let $S_{n}$ denote the symmetric group on the numbers $\{1,2, \cdots, n\}$. Define an action of $S_{n}$ on $\Pi(N)$ as follows; for $\sigma \in S_{n}$ and $B_{1} / \cdots / B_{k} \in$ $\Pi(N)$

$$
\left(B_{1} / \cdots / B_{k}\right) \sigma=B_{1} \sigma / B_{2} \sigma / \cdots / B_{k} \sigma
$$


where $B_{i} \sigma=\left\{b \sigma: b \in B_{i}\right\}$. It is easily checked that this permutation representation is faithful and that each $\sigma \in S_{n}$ acts as an automorphism of $\Pi(N)$.

Recall that a lattice $L$ is upper semimodular provided that all pairs of elements $x, y \in L$ satisfy the condition $\left({ }^{*}\right)$ :

$\left(^{*}\right) \quad$ If $x$ and $y$ both cover $x \wedge y$ then $x \vee y$ covers both $x$ and $y$.

A lattice $G$ is geometric if it is upper semimodular and if each element of $G$ is a join of atoms. Its easy to check that every finite partition lattice is geometric.

Let $L$ be a finite lattice and $\Delta$ a maximal chain in $L$ from 0 to 1. If, for every chain $K$ of $L$ the sublattice of $L$ generated by $K$ and $\Delta$ is distributive, then we call $\Delta$ an $M$-chain of $L$ and we call $(L, \Delta)$ a supersolvable lattice (SS-lattice).

Let $L$ be a finite lattice with rank function $r$ and let $m=r(1)$. The Birkhoff polynomial of $L$, denoted $B_{L}(\lambda)$ is defined by

$$
B_{L}(\lambda)=\sum_{x \in L} \mu(0, x) \lambda^{m-r(x)} .
$$

Here $\mu$ is the usual Möbius function of $L$.

It is assumed in $\S \S 3$ and 5 that the reader is familiar with the structure theory for supersolvable lattices given by Stanley and particularly with his elegant results concerning Birkhoff polynomials of supersolvable geometric lattices (see Stanley [4]). For more about lattice theory see Dilworth and Crawley, [2].

If $K$ is a lattice and $S$ a subset of $K$ we say $S$ is a meet-sublattice of $K$ if $S$ together with the inherited ordering is a lattice in which the meet agrees with the meet in $K$.

2. The structure of $(\Pi(N))_{\sigma^{*}}$ Throughout this section we assume that $n$ is a fixed positive integer and that $\sigma$ is a permutation of $\{1,2, \cdots, n\}$. We write

$$
\sigma=\left(c_{1,1}, \cdots, c_{1, l_{1}}\right) \cdots\left(c_{m, 1}, \cdots, c_{m, l_{m}}\right)
$$

according to its disjoint cycle decomposition as a permutation of $\{1,2, \cdots, n\}$. We refer to $\left(c_{i, 1}, \cdots, c_{i, l_{i}}\right)$ as the $i$ th cycle of $\sigma$ and denote it by $C_{i}$. Note that $l_{i}$ is the length of $C_{i}$ and so $l_{1}+\cdots+$ $l_{m}=n$.

Let $L$ denote the fixed point partition lattice $(\Pi(N))_{\sigma}$. Observe that if $\beta=B_{1} / \cdots \mid B_{k} \in L$ then $B_{1} / \cdots\left|B_{k}=B_{1} \sigma\right| \cdots \mid B_{k} \sigma$ and so $\sigma$ permutes the blocks of $\beta$. We let $Z(\sigma ; \beta)$ denote the cycle indicator of this induced action of $\sigma$ on the set of blocks of $\beta$. The following observation is presented without proof. 
Lemma 1. Suppose $\beta=B_{1} / \cdots / B_{k} \in L$ and $m_{s, u} \in B_{i_{0}}$. Then there exists an integer $d$ which divides $l_{\mathrm{s}}$ and there exist distinct blocks $B_{i_{0}}, B_{i_{1}}, \cdots, B_{i_{d-1}}$ such that the elements of the cycle $C_{s}$ are evenly divided amongst the $d$ blocks $B_{i_{0}}, \cdots, B_{i_{d-1}}$ according to the rule

$$
m_{s, t} \in B_{i_{r}} \quad \text { iff } \quad u-t \equiv r \quad \bmod \left(l_{s} / d\right) .
$$

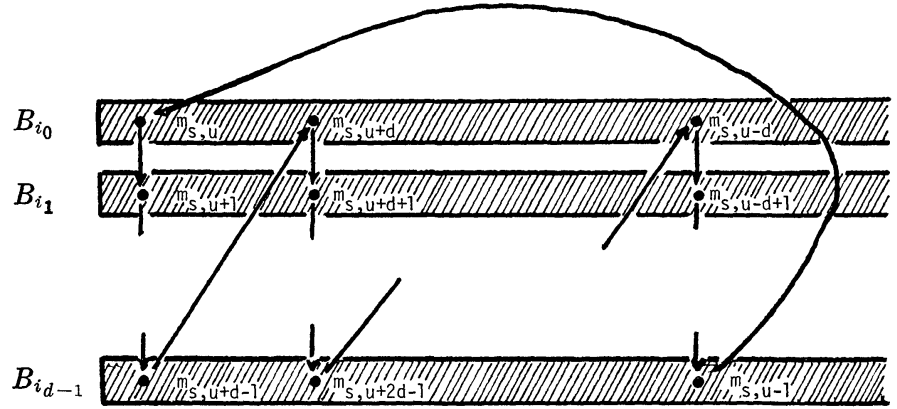

FiguRE 1

In a similar way, $\beta$ induces a partition of the set of cycles $\left\{C_{1}, \cdots, C_{m}\right\}$ which is defined in terms of the equivalence relation $\sim$ by $C_{i} \sim C_{j}$ iff there exists $c \in C_{i}, d \in C_{j}$ and a block of $\beta$ containing both $c$ and $d$. This relation is transitive since each cycle is divided amongst a cyclically permuted set of blocks. We denote the resulting partition of $\left\{C_{1}, \cdots, C_{m}\right\}$ by $\rho(\sigma ; \beta)$.

EXAMPLE 1. Let $n=4$ and $\sigma=(1,2)(3,4)$. The partition $\beta=$ $1 / 2 / 34$ is in $L$; the cycle indicator $Z(\sigma ; \beta)=x_{1} x_{2}$ and the partition $\rho(\sigma ; \beta)$ puts each cycle in a block by itself.

If instead we let $\beta=13 / 24$ we have $Z(\sigma ; \beta)=x_{2}$ whereas the partition $\rho(\sigma ; \beta)$ has just one block containing the two cycles. The lattice $L$ appears in the figure below.

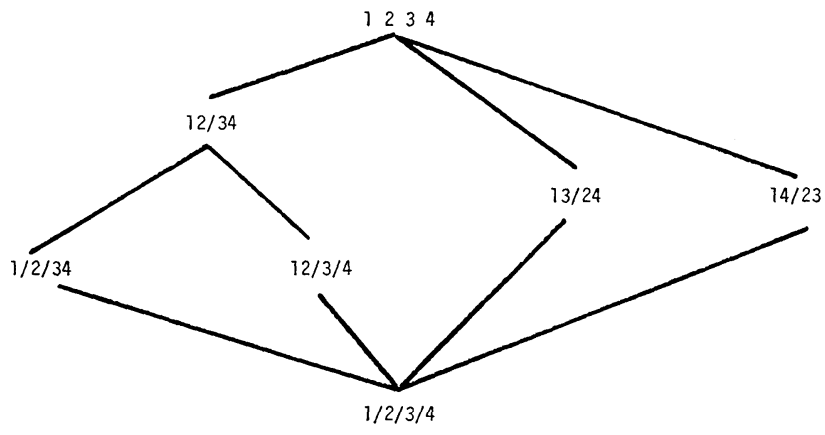

Figure 2 
Note that $L$ is not Jordan; in general the fixed point lattices $(\Pi(N))_{\sigma}$ are not themselves highly structured. However the meet sublattice $\mathscr{L}$ of $L$ consisting of 1 together with all meets of coatoms in $L$ is highly structured, in the above case isomorphic to the lattice of partitions of a 3 element set. We begin by investigating the coatoms of $L$.

LEMma 2. There are two kinds of coatoms $\gamma$ in $L$ :

(a) $\gamma$ has 2 blocks, $\gamma=B_{1} / B_{2}$. Each block is setwise invariant under $\sigma$ hence each block is a union of cycles. $Z(\sigma, \gamma)=x_{1}^{2}$ and $\rho(\sigma, \gamma)$ is a coatom in the lattice of partitions of $\left\{C_{1}, \cdots, C_{m}\right\}$.

(b) $\gamma$ has $p$ blocks, $\gamma=B_{1} / \cdots / B_{p}$, where $p$ is a prime. The blocks $B_{p}$ are cyclically permuted by $\sigma$ and every cycle $C_{i}$ is divided evenly amongst the blocks $B_{1}, \cdots, B_{p}$. The integer $p$ divides $\operatorname{gcd}\left(l_{1}, \cdots, l_{m}\right), Z(\sigma, \gamma)=x_{p}$ and $\rho(\sigma, \gamma)$ is the 1 in the lattice of partitions of $\left\{C_{1}, \cdots, C_{m}\right\}$.

Proof. Clearly each of the 2 sorts of partitions above is fixed by $\sigma$ and each is a coatom in $L$.

Let $\gamma$ be a coatom of $L$ where $\gamma=B_{1} / \cdots / B_{k}(k \geqq 2)$. Suppose the blocks of $\gamma$ can be split into two disjoint $\sigma$-invariant sets

$$
\begin{aligned}
& S=\left\{B_{i_{1}}, \cdots, B_{i_{u}}\right\} \\
& T=\left\{B_{j_{1}}, \cdots, B_{j_{v}}\right\} .
\end{aligned}
$$

Consider the partition $\gamma^{\prime}=\left(\bigcup_{B_{i} \in S} B_{i}\right) /\left(\bigcup_{B_{j} \in T} B_{j}\right)$. Clearly $\gamma^{\prime} \in L$ and $\gamma \leqq \gamma^{\prime}<1$. As $\gamma$ is a coatom of $L, \gamma^{\prime}=\gamma$ and so $u=v=1$. Thus $\gamma$ is of type (a).

Otherwise, $\sigma$ acts transitively on the set of blocks $\left\{B_{1}, \cdots, B_{k}\right\}$. Assume the $B_{i}$ 's are numbered so that $B_{i} \sigma=B_{i+1}$ for $i<k$ and $B_{k} \sigma=B_{1}$. Suppose $k$ factors as $k=r s$ where $r>1$ and $s \geqq 1$. Consider the partition

$$
\gamma^{\prime}=\left(\bigcup_{i=0}^{s-1} B_{1+r i}\right) /\left(\bigcup_{i=0}^{s-1} B_{2+r i}\right) / \cdots /\left(\bigcup_{i=0}^{s-1} B_{r+r i}\right) .
$$

Clearly $\gamma^{\prime} \in L$ and $\gamma \leqq \gamma^{\prime}<1$, so $\gamma=\gamma^{\prime}$. Thus $s=1$ and $\gamma$ is of type (b).

There are $2^{m-1}-1$ coatoms of the kind outlined in (a); these will be called coatoms of type a. For each prime $p$ dividing $\operatorname{gcd}\left(l_{1}, \cdots, l_{m}\right)$ there are $p^{m-1}$ coatoms of the kind outlined in (b); these will be called coatoms of type $\mathrm{b}$.

Note that the coatoms of type a generate a sublattice of $\mathscr{C}$ isomorphic to the lattice of partitions of $\left\{C_{1}, \cdots, C_{m}\right\}$. In the case 
that $\operatorname{gcd}\left(l_{1}, \cdots, l_{m}\right)=1$ there are no coatoms in $L$ of type $\mathrm{b}$ and so this sublattice is all of $\mathscr{M}$.

A partition $\beta$ in $L$ with $Z(\sigma, \beta)=x_{j}^{i}$ will be called periodic with period $j$. The preceding lemma states that every coatom of $L$ is periodic with period 1 or with prime period. The next lemma will imply that every partition in $\mathscr{M}$ is periodic.

LEMMA 3. Let $\beta_{1}, \beta_{2} \in L$ and suppose $\beta_{1}$ is periodic with period $j_{1}$ and $\beta_{2}$ is periodic with period $j_{2}$. Then $\beta_{1} \wedge \beta_{2}$ is periodic with period $j=\operatorname{lcm}\left(j_{1}, j_{2}\right)$.

Proof. Choose a block $B$ of $\beta_{1} \wedge \beta_{2}$ and let $c_{s, u} \in B$. Applying Lemma 1 and the fact that $\beta_{1}$ has period $j_{1}$ we see that $c_{s, t}$ is in the same block of $\beta_{1}$ as $c_{s, u}$ iff $t \equiv u \bmod \left(l_{s} / j_{1}\right)$. Similarly, $c_{s, t}$ is the same block of $\beta_{2}$ as $c_{s, u}$ iff $t \equiv u \bmod \left(l_{s} / j_{2}\right)$. Hence $c_{s, t}$ is in the same block of $\beta_{1} \wedge \beta_{2}$ iff $t \equiv u \bmod \left(l_{s} / j_{1}\right)$ and $t \equiv u \bmod \left(l_{s} / j_{2}\right)$ iff $t \equiv u$ $\bmod \left(l_{s} / j\right)$ where $j=l c m\left(j_{1}, j_{2}\right)$. Applying Lemma 1 again we have that the block $B$ falls in a $j$-cycle under the action of $\sigma$. As $B$ was chosen arbitrarily we see that every block of $\beta$ falls in a $j$-cycle under the action of $\sigma$ and so $Z(\sigma, \beta)=x_{j}^{i}$.

Write $\operatorname{gcd}\left(l_{1}, \cdots, l_{m}\right)=p_{1}^{a_{1}} \cdots p_{r}^{a_{r}}$ and let $j=p_{1} \cdots p_{r}$. Lemma 3 tells us that every partition in $\mathscr{C}$ has period $i$ where $i / j$. Let $\hat{\sigma}$ be the permutation of $\{1,2, \cdots, m j\}$ which consists of $m$ cycles of length $j$,

$$
\hat{\sigma}=(1,2, \cdots, j)(j+1, \cdots, 2 j) \cdots((m-1) j+1, \cdots, m j) .
$$

Let $\hat{L}$ be the fixed point partition lattice of $\hat{\sigma}$ and let $\hat{\mathscr{C}}$ be the meet sublattice of $\hat{L}$ consisting of 1 together with all meets of coatoms of $\hat{L}$. Let $L$ and $\mathscr{M}$ be as above.

\section{LEMmA 4. The lattices $\mathscr{C l}$ and $\hat{\mathscr{C}}$ are isomorphic.}

Proof. This follows from the classification of coatoms given in Lemma 2. Returning to $\sigma$ note that $c_{1,1}, c_{1, j+1}, c_{1,2 j+1}, \cdots$ are in the same block of every coatom in $L$, and hence they are in the same block of every partition in $\mathscr{C}$. The same is true of $c_{i, k}, c_{i, k+j}, c_{i, k+2 j}, \cdots$ as $i$ ranges from 1 to $m$ and $k$ ranges from 1 to $j$. So there is a natural 1-1 correspondence $\varphi$ between the coatoms of $\hat{\mathscr{C}}$ and the coatoms of $\mathscr{C}$ given as follows; let $\gamma$ be a coatom of $\hat{\mathscr{C}}$ and let $c_{i, k}, c_{r, s} \in\{1,2, \cdots, n\}$. Write $k=j k^{\prime}+u$ and $s=j s^{\prime}+v$ where $1 \leqq$ $u \leqq j$ and $1 \leqq v \leqq j$. Then $c_{i, k}$ and $c_{r, 8}$ are in the same block of $\varphi(\gamma)$ iff $(i-1) j+u$ and $(r-1) j+v$ are in the same block of $\gamma$. 
This is easily seen to be a 1-1 onto mapping between coatoms which extends to a lattice isomorphism between $\hat{\mathscr{C}}$ and $\mathscr{L}$.

In the next section we will study the structure of the lattice $\mathscr{C}$ and in $\S 4$ its associated geometry. By Lemma 4 we may reduce to the case of $\sigma$ having $m$ cycles of length $j$, where $j$ is a product of distinct primes.

5. The supersolvability of $\mathscr{C}$. In this section we study the structure of $\mathscr{C}$. Without loss of generality, we assume that $n=m j$ where $j$ is the product of $r$ distinct primes $j=p_{1} \cdots p_{r}$. We assume that $\sigma$ is the permutation

$$
\sigma=(1,2, \cdots, j)(j+1, \cdots, 2 j) \cdots((m-1) j+1, \cdots, m j)
$$

and as before we call $((i-1) j+1, \cdots, i j)$ the $i$ th cycle of $\sigma$ and denote it $C_{i}$. Since $\sigma$ is fixed we abbreviate $Z(\sigma ; \beta)$ and $\rho(\sigma ; \beta)$ by $Z(\beta)$ and $\rho(\beta)$. Let $L=(\Pi(N))_{\sigma}$ be the fixed point partial ordering of $\sigma$ and let $\mathscr{C}$ be the meet sublattice of $L$ consisting of 1 together with all meets of coatoms.

Let $h$ be the partition in $L$ which puts each cycle in a block by itself:

$$
h=\{1,2, \cdots, j\} /\{j+1, \cdots, 2 j\} / \cdots /\{(m-1) j+1, \cdots, m j\} .
$$

Note that $h$ is the meet of all type a coatoms in $L$ and so $h \in \mathscr{N}$. We call $h$ the hinge of $/ l$.

LEMma 5. In til we have

$$
\begin{aligned}
& {[h, 1] \cong \Pi(M)} \\
& {[0, h] \cong D_{j} \cong B_{r}}
\end{aligned}
$$

where $D_{j}$ denotes the lattice of divisors of $j$ and $B_{r}$ denotes the lattice of subsets of $\{1,2, \cdots, r\}$.

Proof. First consider the interval $[h, 1]$. In $\Pi(N)$, this interval is isomorphic to $\Pi(\{1,2, \cdots, m\})$ and every element of this interval is a meet of coatoms in the interval. Also each partition above $h$ is fixed by $\sigma$ and so $[h, 1] \cong L$. It follows that $[h, 1] \cong \mathscr{L}$ which proves the first assertion.

For the second assertion, recall that each partition in $\mathscr{C l}$ is periodic with period $d$ dividing $j$. For $d \mid j$, there is a unique partition $\tau(d)$ below $h$ of period $d$ consisting of $d m$ blocks. This partition is arrived at by dividing each cycle $C_{i}$ of $\sigma$ into $d$ blocks according to: 


$$
\begin{aligned}
& (i-1) j+s \text { and }(i-1) j+t \text { are in the same block } \\
& \text { iff } s \equiv t \bmod d .
\end{aligned}
$$

If $d=p_{i_{1}} p_{i_{2}} \cdots p_{i_{u}}$ then $\tau(d)$ can be realized as a meet of coatoms in $L$ by taking the meet of all coatoms of type a and one coatom of period $p_{i_{l}}$ for $1 \leqq l \leqq u$. It follows that $[0, h] \cong D_{j}$.

Recall that in a lattice $K$, a complement of an element $k$ is an element $k^{\prime}$ with $k \vee k^{\prime}=1$ and $k \wedge k^{\prime}=0$.

Lemma 6. In the lattice $\mathscr{L}, h$ has $j^{m-1}$ complements, and each complement $c$ has the following properties:

(a) $\rho(c)=1$

(b) $Z(c)=x_{j}^{m}$

(c) $[c, 1] \cong D_{j}$

(d) $[0, c] \cong \Pi(\{1,2, \cdots, m\})$.

Proof. Let $F$ be the set of functions mapping $\{1,2, \cdots, m-1\}$ into the set $\{1,2, \cdots, j\}$, and let $f \in F$. Define a partition $c(f)$ of the set $\{1,2, \cdots, m j\}$ as follows:

(1) The element $(m-1) j+1$ (i.e., the first element in $C_{m}$ ) will be in a block with exactly one element from every other cycle, these $m-1$ elements being $(s-1) j+f(s) s=1,2, \cdots, m-1$.

(2) Rotate this block cyclically under the action of $\sigma$; the element $(m-1) j+i 1 \leqq i \leqq j$ will be in a block with exactly one element from every other cycle, these $m-1$ elements being $(s-1) j+(i+f(s))$ where $1 \leqq s \leqq m-1$ and where $f(s)+i$ is taken $\bmod j$.

It is clear that $c(f)$ uniquely determines $f$ and so there are $j^{m-1}$ such partitions $c(f)$. Note that each has $\rho(c(f))=1$ and $Z(c(f))=x_{j}^{m}$.

Consider the join $h \vee c(f)$ in $\Pi(N)$. In $h$, every pair of elements in a common cycle are in the same block. In $c(f)$, every two cycles have elements in the same block. So $h \vee c(f)=1$.

Next consider the meet $h \wedge c(f)$ in $\Pi(N)$. In $c(f)$, no two elements in the same cycle are in the same block whereas in $h$, no two elements in distinct cycles are in the same block. It follows that $h \wedge c(f)=0$.

So $c(f)$ is a complement to $h$ in $\Pi(N)$ hence $c(f)$ will be a complement to $h$ in $L$. Hence $c(f)$ will be a complement to $h$ in $\mathscr{C}$ provided $c(f)$ is in $\mathscr{C}$. We examine the coatoms in $L$ which sit above $c(f)$; clearly all are of type b. Let $p$ be a prime dividing $j$. Recall that if $\gamma$ is a type $b$ coatom of period $p$ then the element $(m-1) j+1$ is in a block with exactly $(j / p)$ elements from each block $C_{i}$, and specifying any of these elements in $C_{i}$ specifies them 
all. It follows that there is a unique coatom of period $p$ above $c(f)$ for each prime $p$ dividing $j$. The meet of these $r$ coatoms has period $j$ (by Lemma 3) and has the property that $(m-1) j+1$ is in a block with at least one other element from each cycle. Clearly this meet is $c(f)$, and so $c(f) \in \mathscr{M}$. Let the $r$ coatoms above $c(f)$ be labelled $\gamma_{i}, \cdots, \gamma_{r}$ so that $\gamma_{i}$ is the coatom of period $p_{i}$. Define a mapping $\varphi: B_{r} \rightarrow[c(f), 1]$ by $\varphi(\phi)=1, \varphi(S)=\bigwedge_{i \in S} \gamma_{i}$ for $S \neq \varnothing$ (here $[c(f), 1]$ denotes the interval in $\mathscr{C}$ ). Obviously $\varphi(S) \leqq \varphi(T)$ iff $T \subseteq S$, and it is easy to check that $\varphi$ is onto. $\varphi$ is one-to-one by Lemma 3 and the fact that the $p_{i}$ 's are distinct primes. It follows that $[c(f), 1] \cong B_{r} \cong D_{j}$. It is equally simple to show that $[0, c(f)] \cong$ $\Pi(\{1,2, \cdots, m\})$. To obtain the isomorphism $\psi$, recall that $[h, 1] \cong$ $\Pi(\{1,2, \cdots, m\})$. Define $\psi:[h, 1] \rightarrow[0, c(f)]$ by $\psi(x)=c(f) \wedge x$. We've thus shown that $c(f)$ is a complement of $h$ in $M$ having the required properties for each $f \in F$.

It remains to show that every complement of $h$ in $\mathscr{M}$ is of the form $c(f)$ for $f \in F$. Let $c$ be any complement of $h$ in $\mathscr{A l}$. As $h \wedge c=0$, no two elements in a common cycle are in the same block of $c$. As $h \vee c=1$, every cycle must have an element in a block of $c$ with some element of $C_{m}$. By the invariance of $c$ under $\sigma$, we may assume that the block of $c$ containing $(m-1) j+1$ contains exactly one element from every other cycle. It is now clear how to define $f \in F$ with $c(f)=c$.

EXAMPLE 2. Let $m=3$ and $j=2$. So our permutation $\sigma=$ $(1,2)(3,4)(5,6)$. The lattice $\mathscr{C l}$ appears below; note that $\mathscr{K}$ is geo-

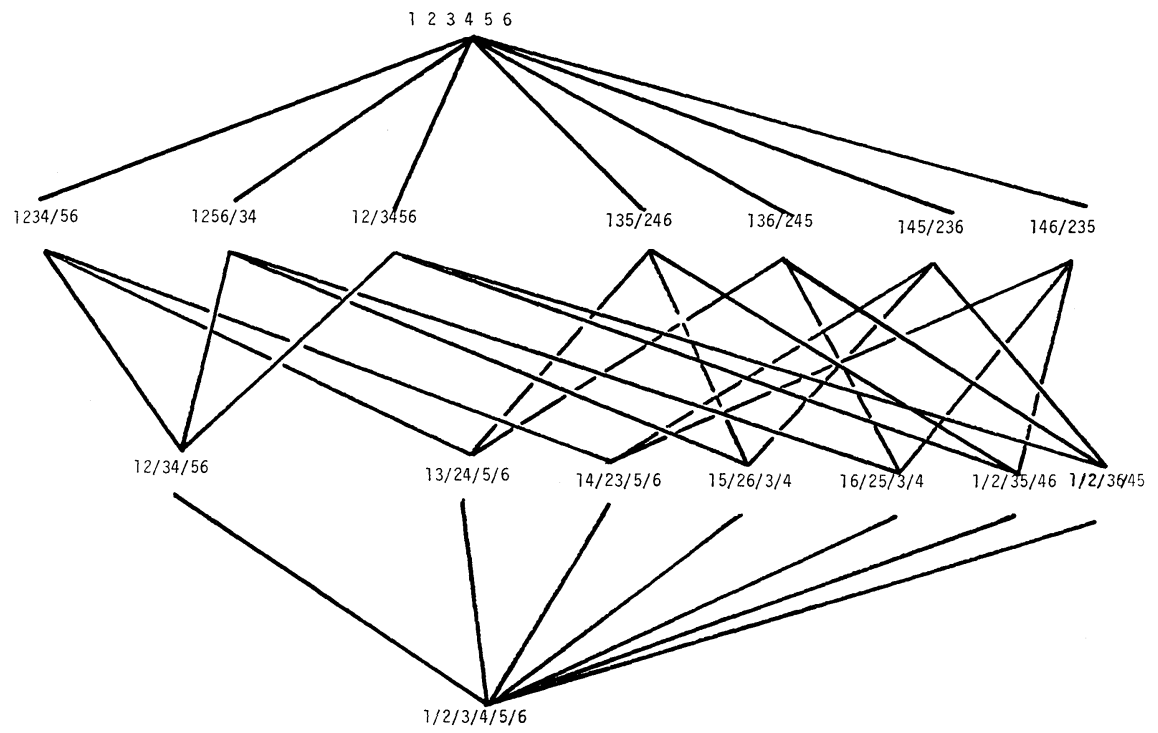

FIGURE 3 
metric. We will see later that $\mathscr{C}$ is geometric iff $j$ is a prime. Here the hinge $h$ is the partition $12 / 34 / 56$. The coatoms of type a are the three to the left, those of type $b$ are the four to the right. $j^{m-1}$ is four; the four complements of $h$ are the four coatoms of type $b$.

In this section we prove that $\mathscr{C}$ is supersolvable. This will require careful analysis of certain elements of $\mathscr{A}$. Recall that if $x \in \mathscr{C}$ then $x$ is periodic of some period $d$ which divides $j$. We let $\Pi(x)$ denote this number $d$. In the following sequence of lemmas, we explore the functions $\Pi$ and $\rho$ and show that a certain miximal chain from 0 to 1 in $\mathscr{C}$ consists of modular elements.

For $x, y \in \mathscr{C l}$ we let $x \vee y$ denote the join of $x$ and $y$ in $\mathscr{K}$ and we let $x \mathrm{~V}_{L} y$ denote the join of $x$ and $y$ in $L . A s \mathscr{M}$ is a meet sublattice of $L$ we have $x \mathrm{~V}_{L} y \leqq x \vee y$; in general equality does not hold. For example, let $j=2$ and $m=3$ so $\sigma=(1,2)(3,4)(5,6)$. Let $x=13 / 24 / 5 / 6$ and let $y=14 / 23 / 5 / 6$. Then $x \bigvee_{L} y=1234 / 5 / 6$ but $x \vee y$ must have period 1 since both $C_{1}$ and $C_{2}$ are in the same block of $x \mathrm{~V}_{L} y$. Hence $x \vee y=1234 / 56$ (see Figure 3).

The function $\rho$, introduced in $\S 2$, is defined for all $x \in L$. It is easy to check that $\rho$ respects the join in $L$, that is $\rho(x) \vee \rho(y)=$ $\rho\left(x \bigvee_{L} y\right)$. In fact $\rho$ also respects the join in $\mathscr{A}$.

Lemma 7. Let $x, y \in \mathscr{M}$. Then $\rho(x \vee y)=\rho(x) \vee \rho(y)$.

Proof. Note that if $\omega, z \in \mathscr{C}$ and $\omega \leqq z$ then $\rho(\omega) \leqq \rho(z)$. So $\rho(x) \vee \rho(y)=\rho\left(x \bigvee_{L} y\right) \leqq \rho(x \vee y)$.

Let $z$ be the unique partition in $\mathscr{C}$ with $\rho(z)=\rho(x) \vee \rho(y)$ and $\Pi(z)=1$. Then $z \geqq x$ and $z \geqq y$ so $x \vee y \leqq z$. Hence $\rho(x \vee y) \leqq$ $\rho(z)=\rho(x) \vee \rho(y)$.

It should be pointed out that the analogous statement for meets is false; i.e., in general we do not have $\rho(x \wedge y)=\rho(x) \wedge \rho(y)$. As a counter example let $j=2$ and $m=2$ so $\sigma=(1,2)(3,4)$. Let $x=$ $13 / 24$ and let $y=14 / 23$. Then $x \wedge y=1 / 2 / 3 / 4$ so $\rho(x \wedge y)=1 / 2$. But $\rho(x)=\rho(y)=12$ so $\rho(x \wedge y)=1 / 2 \neq 12=\rho(x) \wedge \rho(y)$. However one case where equality holds will be of particular interest to us.

Lemma 7. Let $x \in \mathscr{M}$ and suppose $\Pi(x)=1$. For any $y \in \mathscr{A}$, $\rho(x \wedge y)=\rho(x) \wedge \rho(y)$.

Proof. As $\Pi(x)=1$, each cycle $C_{i}$ is contained in a block of $x$. Let $C_{p}$ and $C_{q}$ be cycles with $p$ and $q$ in the same block of $\rho(x) \wedge \rho(y)$. Then $p$ and $q$ lie in the same block of $\rho(y)$ so there exist $u \in C_{p}$ and $v \in C_{q}$ such that $u$ and $v$ lie in the same block of $y$. Also $p$ and $q$ lie in the same block of $\rho(x)$ so some block of $x$ contains both cycles 
$C_{p}$ and $C_{q}$. Hence $a$ and $v$ lie in the same block of $x \wedge y$ so $p$ and $q$ lie in the same block of $\rho(x \wedge y)$. This shows that $\rho(x) \wedge \rho(y) \leqq$ $\rho(x \wedge y)$; the reverse inequality is easy to show.

We next consider the function $\Pi$. Again we will be interested in how it behaves with respect to the join operation in $\mathscr{l}$.

Lemma 9. Let $x, y \in \mathscr{A}$.

(A) If $x \leqq y$ then $\Pi(y) \mid \Pi(x)$.

(B) $\Pi(x \vee y)$ divides ged $(\Pi(x), \Pi(y))$.

(C) If $\Pi(x \vee y)=\operatorname{gcd}(\Pi(x), \Pi(y))$ then $x \vee y=x \bigvee_{L} y$.

Proof. Note that $\Pi(x)=d$ iff the elements of each cycle $C_{i}$ are evenly divided amongst $d$ blocks according to the rule that $u$ and $v$ are in the same block iff $u \equiv v(\bmod d)$, for $u, v \in C_{i}$. From this observation (A) follows immediately, and (B) follows easily from (A).

For (c) suppose first that $u, v \in C_{i}$ and $u \equiv v(\bmod \operatorname{gcd}(d, e))$ : say $u=v+k \operatorname{gcd}(d, e)$. Write $k \operatorname{gcd}(d, e)=\alpha d+\beta e$ for $\alpha, \beta \in Z$ and let $\omega$ be the unique element of $C_{i}$ satisfying $u+\alpha d \equiv \omega(\bmod j)$. Then $u$ and $\omega$ are equivalent $\bmod d$ hence are in the same block of $x$. Also

$$
\omega+\beta e=(u+\alpha d)+\beta e=u+k \operatorname{gcd}(d, e)=v
$$

so $w$ and $v$ are equivalent $\bmod e$ hence are in the same block of $y$. Thus $u$ and $v$ are in the same block of $x \mathrm{~V}_{L} y$, which shows that if $u \equiv v(\bmod \operatorname{gcd}(\Pi(x), \Pi(y)))$ and $u, v \in C_{i}$ then $u$ and $v$ are in the same block of $x \mathrm{~V}_{L} y$.

Suppose $u$ and $w$ are in the same block of $x \vee y$ with $u \in C_{p}$ and $w \in C_{q}$. Since

$$
\rho(x \vee y)=\rho(x) \vee \rho(y) \text { and } \rho(x) \vee \rho(y)=\rho(x \underset{L}{\bigvee} y)
$$

there exists a sequence $u=u_{0}, u_{1}, \cdots, u_{n}$ such that $u_{i}, u_{i+1}$ are in the same block of either $x$ or $y$ and such that $u_{n} \in C_{q}$. It follows that $u$ and $u_{n}$ are in the same block of $x \bigvee_{L} y$ hence of $x \vee y$ so $w$ and $u_{n}$ are in the same cycle and in the same block of $x \vee y$. So $u_{n}-w \equiv 0$ $(\bmod \Pi(x \vee y))$. Since $\Pi(x \vee y)=\Pi\left(x \vee_{L} y\right)$ we see that $u_{n} \equiv w$ $\left(\bmod \Pi\left(x \mathrm{~V}_{L} y\right)\right.$. By the above observation, $u_{n}$ and $w$ (hence $u$ and $w)$ are in the same block of $x \bigvee_{L} y$ so $x \vee y \leqq x \bigvee_{L} y$ and equality must hold.

Note that the sufficient condition for the equality of $x \vee y$ and $x \mathrm{~V}_{L} y$ given in (C) is not a necessary condition. For a counterexample let $j=2$ and $m=4$ so $\sigma=(1,2)(3,4)(5,6)(7,8)$. Let $x=$ $14 / 23 / 58 / 67$ and let $y=13 / 24 / 57 / 68$. Then

$$
x \vee y=x \underset{L}{\bigvee} y=1234 / 5678 \text { so } \Pi(x \vee y)=1
$$


But $\Pi(x)=\Pi(y)=2$ so $2=\operatorname{gcd}(\Pi(x), \Pi(y))$.

We can now construct the bottom half of our maximal chain of modular elements. Suppose $\rho(x)=0$ and $\Pi(x)=d$. Then each block of $x$ contains $j / d$ elements; the blocks partition each cycle $C_{i}$ into $d$ parts. The unique element $x$ of $\mathscr{M}$ satisfying these conditions is denoted $\tau(d)$. Note that $\tau(j)=0$ and $\tau(\overline{1})=h$.

LeMma 10 . Let $d / j$ and let $y, z \in \mathscr{M}$.

(A) If $z \leqq y$ then $z \vee(\tau(d) \wedge y)=(z \vee \tau(d)) \wedge y$.

(B) If $z \leqq \tau(d)$ then $z \vee(\tau(d) \wedge y)=(z \vee y) \wedge \tau(d)$.

Proof. We first prove (A). Note that for any $x \in \mathscr{L}, \tau(d) \wedge x=$ $\tau(e)$ where $e=l c m(d, \Pi(x))$ and $\tau(d) \vee x$ is the unique element of $\mathscr{C l}$ above $x$ which has period ged $(d, \Pi(x))$ and cycle partition $\rho(x)$. From this it follows that $z \vee(\tau(d) \wedge y)$ is the unique element of $\mathscr{C}$ above $z$ which satisfies

$$
\begin{aligned}
& \rho(z \vee(\tau(d) \wedge y))=\rho(z) \\
& \Pi(z \vee(\tau(d) \wedge y))=\operatorname{gcd} \Pi(z), \operatorname{lcm}(d, \Pi(y))
\end{aligned}
$$

By a similar argument one shows that $(z \vee \tau(d)) \wedge y$ is the unique element of $\mathscr{C}$ above $z$ which satisfies

$$
\begin{aligned}
& \rho((z \vee \tau(d)) \wedge y)=\rho(z) \\
& \Pi((z \vee \tau(d)) \wedge y)=\operatorname{lcm}(\Pi(y), \operatorname{gcd}(\Pi(z), d)) .
\end{aligned}
$$

Here one needs to use the fact that $z \leqq y$.

As $z \leqq y$ we have $\Pi(y) \mid \Pi(z)$. Also, the lattice of divisors of $j$ is modular which together with $\Pi(y) \mid \Pi(z)$ gives

$$
l c m(\Pi(y), \operatorname{gcd}(\Pi(z), d))=\operatorname{gcd}(\Pi(z), \operatorname{lcm}(d, \Pi(y))) .
$$

The proof of (B) is somewhat easier. Assume $z=\Pi(e)$ where $d \mid e$. Then

$$
\begin{aligned}
z \vee(\tau(d) \wedge y) & =\tau(e) \vee(\tau(d) \wedge y) \\
& =\tau(\operatorname{lcm}(e, \operatorname{gcd}(d, \Pi(y)))) . \\
(z \vee y) \wedge \tau(d) & =(\tau(e) \vee y) \wedge \tau(d) \\
& =\tau(\operatorname{gcd}(d, \operatorname{lcm}(e, \Pi(y)))) .
\end{aligned}
$$

As before, the condition $d \mid e$ together with the modularity of the lattice of divisors of $j$ proves the desired equality.

Recall that $j$ was assumed to be the product of $r$ distinct primes $j=p_{1} p_{2} \cdots p_{r}$. For $i=1,2, \cdots, r$ let $t_{i}=\tau\left(p_{1} p_{2} \cdots p_{i}\right)$, and let $t_{0}=0$. Then $0=t_{0}<t_{1}<\cdots<t_{r}=h$ is a maximal chain from 0 to $h$ consisting of modular elements of $\mathscr{C}$ (by Lemma 10).

For $i=1,2, \cdots, m$ let $s_{i}$ denote the element of $\mathscr{C}$ which has 
the following $i+1$ blocks; block 1 contains only cycle $C_{1}$, block 2 contains only cycle $C_{2}, \cdots$, block $i$ contains only cycle $C_{i}$ and block $i+1$ contains the remaining cycles $C_{i+1}, \cdots, C_{m}$. Let $s_{0}=1$ so

$$
h=s_{m-1}<s_{m-2}<\cdots<s_{0}=1
$$

is a maximal chain from $h$ to 1 . Note that $\Pi\left(s_{i}\right)=1$ and $\rho\left(s_{i}\right)=$ $\{1\} /\{2\} / \cdots /\{i\} /\{i+1, i+2, \cdots, m\}$. We will use the fact that $\rho\left(s_{i}\right)$ is a modular element of $\Pi(M)$.

Lemma 11. Let $y, z \in \mathscr{M}$. For $i=0,1, \cdots, m-1$ we have the following:

(A) If $z \leqq y$ then $z \vee\left(s_{i} \wedge y\right)=\left(z \wedge s_{i}\right) \wedge y$.

(B) If $z \leqq s_{i}$ then $z \vee\left(s_{i} \wedge y\right)=(z \vee y) \wedge s_{i}$.

Proof. We first prove (A); assume $z \leqq y$.

$$
\begin{aligned}
\rho\left(z \vee\left(s_{i} \wedge y\right)\right) & =\rho(z) \vee \rho\left(s_{i} \wedge y\right) & \text { by Lemma } 7 \\
& =\rho(z) \vee\left(\rho\left(s_{i}\right) \wedge \rho(y)\right) & \text { by Lemma } 8 \\
& =\left(\rho(z) \vee \rho\left(s_{i}\right)\right) \wedge \rho(y) &
\end{aligned}
$$

the last equality holding since $\rho\left(s_{i}\right)$ is a modular element of $\Pi(M)$. Using Lemma 7 again we have

$$
\rho\left(z \vee\left(s_{i} \wedge y\right)\right)=\rho\left(z \vee s_{i}\right) \wedge \rho(y)=\rho\left(\left(z \vee s_{i}\right) \wedge y\right)
$$

The last equality follows from Lemma 8 upon observing that $z \vee s_{i} \geqq s_{i}$ so $\Pi\left(z \vee s_{i}\right) \mid \Pi\left(s_{i}\right)=1$.

Also $\Pi\left(s_{i}\right)=\Pi\left(s_{i} \vee z\right)=1$ so $\Pi\left(\left(s_{i} \vee z\right) \wedge y\right)=\Pi(y)$ and $\Pi\left(s_{i} \wedge y\right)=$ $\Pi(y)$. The latter equality implies that $\Pi\left(z \vee\left(s_{i} \wedge y\right)\right) \mid \Pi(y)$. But $y \geqq z$ and $y \geqq s_{i} \wedge y$ so $y \geqq z \vee\left(s_{i} \wedge y\right)$ hence $\Pi(y) \mid \Pi\left(z \vee\left(s_{i} \wedge y\right)\right)$. Thus

$$
\Pi\left(z \vee\left(s_{i} \wedge y\right)\right)=\operatorname{gcd}\left(\Pi(z), \Pi\left(s_{i} \wedge y\right)\right)
$$

and so $z \vee\left(s_{i} \wedge y\right)=z \bigvee_{L}\left(s_{i} \wedge y\right)$ by Lemma $9(\mathrm{C})$. We now show that $z \vee\left(s_{i} \wedge y\right) \leqq\left(s_{i} \vee z\right) \wedge y$ which will imply equality since we know

and

$$
\rho\left(z \vee\left(s_{i} \wedge y\right)\right)=\rho\left(\left(s_{i} \vee z\right) \wedge y\right)
$$

$$
\Pi\left(z \vee\left(s_{i} \wedge y\right)\right)=\Pi\left(\left(s_{i} \vee z\right) \wedge y\right) .
$$

Suppose $u$ and $v$ are in the same block of $z \vee\left(s_{i} \wedge y\right)$. Since $z \vee\left(s_{i} \wedge y\right)=z \bigvee_{L}\left(s_{i} \wedge y\right)$ there exists a sequence $u=u_{0}, u_{1}, \cdots, u_{n}=v$ such that $u_{l}, u_{l+1}$ are in the same block of either $z$ or $\left(s_{i} \wedge y\right)$. Since $z \leqq y$ we see that $u_{l}, u_{l+1}$ are in the same block of $y$ so $u$ and $v$ are in the same block of $y$. Also $u_{l}, u_{l+1}$ are in the same block of either $z$ or $s_{i}$ so $u$ and $v$ are in the same block of $z \bigvee_{L} s_{i}$ hence of 
$z \vee s_{i}$. Thus $u$ and $v$ are in the same block of $\left(z \vee s_{i}\right) \wedge y$ so $(z \vee y) \leqq$ $\left(s_{i} \vee z\right) \wedge y$. This completes the proof of (A).

The proof of (B) is the same with a minor exception. As in (A) we show that

and

$$
\rho\left(z \vee\left(s_{i} \wedge y\right)\right)=\rho\left((z \vee y) \wedge s_{i}\right)
$$

$$
\Pi\left(z \vee\left(s_{i} \wedge y\right)\right)=\Pi(y \vee z)=\Pi\left((z \vee y) \wedge s_{i}\right) .
$$

Let $d=\Pi(z \vee y)$, and suppose that $u$ and $v$ are in the same block of $z \vee\left(s_{i} \wedge y\right)$. Then there exists a sequence $u=u_{0}, u_{1}, \cdots, u_{n}$ such that

(1) $u_{l}, u_{l+1}$ are in the same block of either $z$ or $\left(s_{i} \wedge y\right)$

(2) $u_{n} \equiv v(\bmod d)$.

Note that $u_{l}, u_{l+1}$ are in the same block of $(z \vee y) \wedge s_{i}$ and $\Pi\left((z \vee y) \wedge s_{i}\right)=d$ so $u$ and $v$ are in the same block of $(z \vee y) \wedge s_{i}$. This completes the proof of $(B)$.

Lemma 11 tells us that each $s_{i}$ is a modular element of $\mathscr{M}$. Combining Lemma 10, Lemma 11 and Proposition 2.1 from Stanley [4, pg. 203] gives the following theorem.

THEOREM 1. $\mathscr{M}$ is a supersolvable lattice with M-chain

$$
0=t_{0}<t_{1}<\cdots<t_{r}=h=s_{m-1}<s_{m-2}<\cdots<s_{0}=1 .
$$

At this point a rough sketch of $\mathscr{C l}$ is helpful.

4. The geometric properties of $\mathscr{M}$. Figure 4 suggests that $\mathscr{C}$ might be geometric; in fact $\mathscr{C}$ is geometric iff $j$ is prime. However $\mathscr{C l}$ does give rise to a pregeometry (in the language of Crapo and Rota [1]) which we will show in this section. To do so

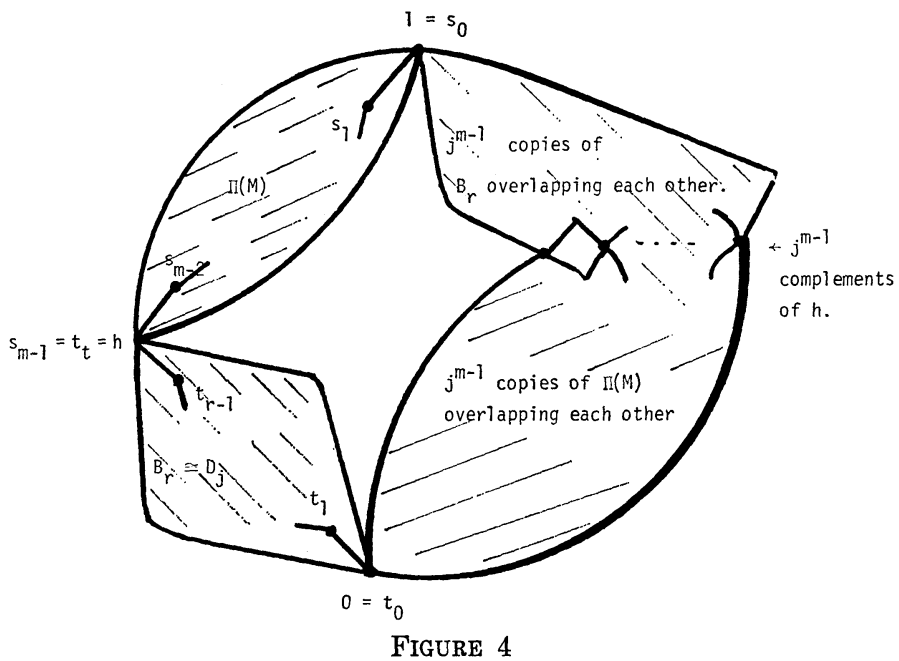


we need notation for certain elements of $\mathscr{C}$. Some of this notation has already been established; for completeness it is listed below again.

(1) For $d \mid j, \tau(d)$ denotes the unique element of ./l with $\rho(\tau(d))=0$ and $\Pi(\tau(d))=d . \quad \tau(d)$ sits in the interval $[0, h]$.

(2) For a partition $\beta \in \Pi(\mathscr{C}), \sigma(\beta)$ denotes the unique element of $\mathscr{l}$ with $\rho(\sigma(\beta))=\beta$ and $\Pi(\sigma(\beta))=1 . \quad \sigma(\beta)$ sits in the interval $[h, 1]$.

(3) Let $F$ be the set of functions mapping $\{1,2, \cdots, m-1\}$ into the set $\{1,2, \cdots, j\}$. For $f \in F, c(f)$ denotes the complement of $h$ given by $f$ as in the proof of Lemma 6 . Note: for notational convenience in what follows we will extend $f$ to a function from $\{1,2, \cdots, m\}$ into $\{1,2, \cdots, j\}$ by defining $f(m)=1$.

(4) Let $p$ and $q$ be integers between 1 and $m$ with $p<q$ and let $r$ be an integer between 0 and $j-1$. Then $\alpha(p, q, r)$ denotes the following partition in $/ \mathbb{C}$ which has exactly $j$ blocks of size 2 and all other blocks of size 1 . Each block of size 2 consists of one element from $C_{p}$ and one from $C_{q}$ according to $u \in C_{p}$ and $v \in C_{q}$ are in the same block iff $u \equiv v-r(\bmod j)$.

ExAmple 3 . Let $j=m=3$ so $\sigma=(1,2,3)(4,5,6)(7,8,9)$. Let $p=1, q=3$ and $r=2$. Then

$$
\alpha(1,3,2)=19 / 27 / 38 / 4 / 5 / 6 .
$$

It is worth noting that $\Pi(\alpha(p, q, r))=j$ and that $\rho(\alpha(p, q, r))$ is the atom in $\Pi(\mathscr{C})$ having the block $\{p, q\}$ of size 2 and all other blocks of size 1 .

LeMma 12. Tl has exactly $r+j\left(\begin{array}{c}m \\ 2\end{array}\right)$ atoms. Of these, $r$ atoms lie in the interval $[0, h]$; these are of the form $\tau(j / p)$ for $p$ a prime dividing $j$. (These $r$ atoms will be called type a atoms.) The remaining $j\left(\begin{array}{c}m \\ 2\end{array}\right)$ atoms lie outside the interval $[0, h]$. These are of the form $\alpha(p, q, r)$ and will be called type $\mathrm{b}$ atoms.

Proof. Let $x$ be an atom. It is clear that $\rho(x)$ is either 0 or an atom in $\Pi(\mathscr{C})$ and that $\Pi(x)$ is either $j$ or $(j / p)$ for $p$ a prime dividing $j$. We consider the four possibilities.

If $\rho(x)=0$ and $\Pi(x)=j$ then $x=0$ which is impossible. If $\rho(x)=0$ and $\Pi(x)$ is $j / p$ then $x=\tau(j / p)$. If $\rho(x)$ is an atom and $\Pi(x)$ is $j / p$ then we have $0<\tau(j / p)<x$ which is impossible.

Lastly suppose $\Pi(x)=j$ and $\rho(x)$ is the atom in $\Pi(\mathscr{l})$ which has exactly one block of size 2 containing $p$ and $q$ with $p<q$. Consider $(p-1) j+1 \in C_{p}$. It is in a block of size 2 with a unique 
element of $C_{q}$, say $(q-1) j+(r+1)$ for $0 \leqq r \leqq j-1$. It is now clear that $x=\alpha(p, q, r)$.

For the remainder of this paper, $A$ denotes the set of type a atoms and $B$ denotes the set of type b atoms. Let $\beta \in \Pi(M)$ and let $f \in F$. Then $B(\beta)$ denotes the set of type b atoms $x$ satisfying $x \leqq \sigma(\beta)$ and $B(f)$ denotes the set of type b atoms satisfying $x \leqq c(f)$. $B(\beta ; f)$ denotes the intersection of $B(\beta)$ and $B(f)$. Note that $\alpha(p, q, r)$ is in $B(\beta)$ iff $p$ and $q$ are in the same block of $\beta$ and $\alpha(p, q, r)$ is in $B(f)$ iff $r \equiv f(q)-f(p)(\bmod j)$.

Let $\mathscr{B}$ denonte the lattice of subsets of $A \cup B$.

Definition 2. Define closure operator ${ }^{-}$on $\mathscr{B}$ as follows; let $S \in \mathscr{B}$ and write $S=S_{A} \cup S_{B}$ with $S_{A} \subseteq A$ and $S_{B} \subseteq B$. Let $\beta=$ $\mathrm{V}_{x \in S_{B}} \rho(x) \in \Pi(M)$. Then

Case 1. $\bar{\phi}=\varnothing$

Case 2. If $S_{A}=\varnothing \neq S_{B}$ and if there exists $f \in F$ such that $x \leqq c(f)$ for all $x \in S_{B}$ let $\bar{S}=B(\beta ; f)$.

Case 3. Let $\bar{S}=A \cup B(\beta)$ otherwise.

We need to show that - is well-defined in Case 2. Suppose $S_{A}=\varnothing \neq S_{B}$ and let $f, g \in F$ satisfy $x \leqq c(f)$ and $x \leqq c(g)$ for all $x \in S_{B}$. We need to show that $B(\beta ; f)=B(\beta ; g)$. By the symmetry of $f$ and $g$ it suffices to prove that $B(\beta ; f) \subseteq B(\beta ; g)$.

Assume that $\alpha(p, q, r) \in B(\beta, f)$ so $r \equiv f(q)-f(p) \bmod j$. Choose a sequence $\alpha\left(p_{0}, p_{1}, r_{1}\right), \alpha\left(p_{1}, p_{2}, r_{2}\right), \cdots, \alpha\left(p_{n-1}, p_{n}, r_{n}\right) \in S_{B}$ such that $p=p_{0}$ and $q=p_{n}$. This can be done by definition of $\beta$. As $x \leqq c(f)$ for all $x \in S_{B}$ we know

$$
f\left(p_{l}\right)-f\left(p_{l-1}\right) \equiv r_{l} \quad(\bmod j) .
$$

In particular

$$
r \equiv f(q)-f(p) \equiv f\left(p_{n}\right)-f\left(p_{0}\right) \equiv \sum_{l=1}^{n}\left(f\left(p_{l}\right)-f\left(p_{l-1}\right)\right) \quad(\bmod j) .
$$

Hence $r \equiv \sum_{l=1}^{n} r_{l}(\bmod j)$. Since $x \leqq c(g)$ for all $x \in S_{B}$ we also have $r_{l} \equiv g\left(p_{l}\right)-g\left(p_{l-1}\right)(\bmod j)$. The same telescoping sum shows that

$$
r \equiv g\left(p_{n}\right)-g\left(p_{0}\right) \equiv g(q)-g(p) \quad(\bmod j)
$$

and so $\alpha(p, q, r) \in B(\beta ; g)$ as desired.

It is easy to show that ${ }^{-}$is a closure operator-the verification is left to the reader. The next lemma shows that - also satisfies the exchange condition thus making $\left(\beta,{ }^{-}\right)$into a pregeometry. We 
first need the following technical lemma.

Lemma 13. Let $S_{B} \subseteq B$ and let $y \in B$. Let $\beta=\bigvee_{z \in S_{B}} \rho(z)$ and suppose that $\bar{S}_{B}$ is of the form $B(\beta ; f)$ whereas $\overline{S_{B} \cup\{y\}}$ is of the form $A \cup B(\gamma)$ for some $\gamma \geqq \beta$. Then $\rho(y) \leqq \beta$ and so $\gamma=\beta$.

Proof. Suppose $\rho(y) \not \beta$. We will construct a function $g \in F$ with $y \leqq c(g)$ and $z \leqq c(g)$ for all $z \in S_{B}$. Let $y=\alpha(p, q, r)$. As $\rho(y) \nsubseteq \beta$ we know that $p$ and $q$ lie in distinct blocks of $\beta$. Write

$$
\beta=B_{1} / B_{2} / \cdots / B_{k} \quad \text { with } p \in B_{1} \text { and } q \in B_{2} .
$$

Case 1. $\quad m \notin B_{1}$. Define $g(l)=f(l)$ for $l \notin B_{1}$.

For $l \in B_{1}$ define

$$
g(l) \equiv(f(q)-f(p))-r+f(l) \quad(\bmod j) .
$$

Note that $g(p) \equiv f(q)-r=g(q)-r(\bmod j)$. Thus $g(q)-g(p) \equiv r$ $(\bmod j)$ and so $y \leqq c(g)$. Suppose $z \in S_{B}, z=\alpha\left(p_{1}, q_{1}, r_{1}\right)$. If $p_{1}, q_{1} \in B_{i}$ for $i \neq 1$ then $g\left(q_{1}\right)-g\left(p_{1}\right) \equiv f\left(q_{1}\right)-f\left(p_{1}\right) \equiv r_{1}(\bmod j)$ and so $z<c(g)$. If $p_{1}, q_{1} \in B_{1}$ then

$$
\begin{aligned}
g\left(q_{1}\right)-g\left(p_{1}\right) & \equiv\left(f(q)-f(p)-r+f\left(q_{1}\right)\right)-\left(f(q)-f(p)-r+f\left(p_{1}\right)\right) \\
& \equiv f\left(q_{1}\right)-f\left(p_{1}\right) \equiv r_{1} \quad(\bmod j) .
\end{aligned}
$$

So $z \leqq c(g)$ as was to be shown.

Case 2. $m \in B_{1}$. Define $g(l)=f(l)$ for $l \notin B_{2}$. For $l \in B_{2}$ define

$$
g(l) \equiv f(l)+(f(p)-f(q))+r \quad(\bmod j) .
$$

As before, $g(q) \equiv f(p)+r=g(p)+r(\bmod j)$ so $y \leqq c(g)$. For $z \in S_{B}$, $z \leqq c(g)$ as in Case 1 .

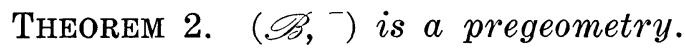

Proof. We need to show that ${ }^{-}$satisfies the following exchange property $(*)$ :

Let $x, y \in A \cup B$ and let $S \subseteq A \cup B$. If $x \notin \bar{S}$ and $x \in \overline{S \cup\{y\}}$ then $y \in \overline{S \cup\{x\}}$.

The verification of $\left(^{*}\right)$ proceeds in several cases. Let $\beta=\mathbf{V}_{z \in S_{B}} \rho(z)$.

Case 1. $x \in A$.

Since $x \notin \bar{S}$ we know $S=S_{B} \subseteq B$. If $y \in A$ then obviously $y \in$ $\overline{S \cup\{x\}}=A \cup B(\beta)$, so assume that $y \in B$. 
Since $x \notin \bar{S}_{B}$, we have $\bar{S}_{B}=B(\beta ; f)$ for some $f \in F . \quad$ As $x \in \overline{S_{B} \cup\{y\}}$ we know $\overline{S_{B} \cup\{y\}}=B(\gamma) \cup A$ for some $\gamma \geqq \beta$. Applying Lemma 13 we have $\rho(y)<\beta$ so $y \in B(\beta)$. So $y \in \overline{S_{B} \cup\{x\}}=B(\beta) \cup A$.

Case 2. $x \in B, y \in A$.

If $y \in \bar{S}$ then

$$
\bar{S} \subseteq \overline{S \cup\{y\}} \subseteq \overline{\bar{S} \cup\{y\}}=\overline{\bar{S}}=\bar{S}
$$

which is impossible since $x \in \overline{S \cup\{y\}}-\bar{S}$.

So $y \notin \bar{S}$; i.e., $\bar{S}=B(\beta ; f)$ for some $f \in F$. Thus $S \cup\{y\}=A \cup B(\beta)$ and so $\rho(x) \leqq \beta$.

Since $x \notin \bar{S}$ there is no function $f \in F$ with $x \leqq c(f)$ and with $z \leqq c(f)$ for all $z \in S$. So $\overline{S \cup\{x\}}=B(\beta) \cup A$ which gives $y \in \overline{S \cup\{x\}}$.

Case 3. $x, y \in B$ and $\rho(y) \leqq \beta$.

Since $\bar{S}$ is properly contained in $\overline{S \cup\{y\}}$ we see that $\bar{S}$ has the form $B(\beta ; f)$ for some $f \in F$ and that $\overline{S \cup\{y\}}=B(\beta) \cup A$. As $x \epsilon$ $\overline{S \cup\{y\}}, \rho(x) \leqq \beta$.

Since $x \notin \bar{S}$ there is no function $f \in F$ with $x \leqq c(f)$ and $z \leqq c(f)$ for all $z \in S$. Thus $S \cup\{x\}=B(\beta) \cup A$ and so $y \in\{x\}$.

Case 4. $\quad x, y \in B, \rho(y) \not \equiv$ and $\bar{S}=A \cup B(\beta)$.

Here we have $\overline{S \cup\{y\}}=A \cup B(\gamma)$ for $\gamma=\beta \vee \rho(y)>\beta$. Since $x \notin \bar{S}$ we know $\rho(x) \leqq \beta$ but $\rho(x) \leqq \beta \vee \rho(y)$. Hence we know $\rho(y) \leqq$ $\beta \vee \rho(x)$ because $\Pi(M)$ is a geometric lattice.

Case 5. $\quad x, y \in B, \rho(y) \not \nexists \beta$ and $S=B(\beta ; f)$ for $f \in F$.

In this case we have $\overline{S \cup\{y\}}=B(\gamma ; g)$ for $\gamma=\beta \vee \rho(y)$ and for some $g \in F$ (see the proof of Lemma 13). Suppose $\rho(x) \leqq \beta$. Since $x \in \overline{S \cup\{y\}}$, we know $x \leqq c(g)$ and so

$$
x \in B(\beta ; g)=B(\beta ; f)=\bar{S} \rightarrow \leftarrow .
$$

Thus $\rho(x) \not \equiv \beta$ and $\rho(x) \leqq \beta \vee \rho(y)$ so $\rho(y) \leqq \beta \vee \rho(x)$ again because $\Pi(\mathscr{C l})$ is geometric. Hence $y \in B(\gamma ; g)=\overline{S \cup\{x\}}$ and this finishes the proof of Theorem 2.

Let $G$ be the subset of $\mathscr{C}$ consisting of all elements of period 1 together with all elements of period $j$. It is clear that if $x, y \in G$ then $x \wedge y \in G$ so $G$ is closed under meets.

Given any element $x$ of $\mathscr{C}$, there is a unique smallest element of period 1 which is greater than or equal to $x$, this being $\sigma(\rho(x))$. In particular this is true of $x=y \vee z$ for $y, z \in G$. Thus $G$ has a join operation $\mathrm{V}_{G}$ defined as follows; for $y, z \in G$

$$
y \bigvee_{G} z=\left\{\begin{array}{lll}
y \vee z & \text { if } & \Pi(y \vee z)=j \\
\sigma(\rho(y \vee z)) & \text { if } & \Pi(y \vee z)<j
\end{array}\right.
$$


$G$ is a meet sublattice of $\mathscr{C}$ hence of $L$ and so of $\Pi(\{1,2, \cdots, m j\})$. For the remainder of the paper we continue to let $\vee, \wedge$ denote the join and meet of $\mathscr{M}$ and $\bigvee_{G}, \Lambda_{G}$ denote the join and meet of $G$.

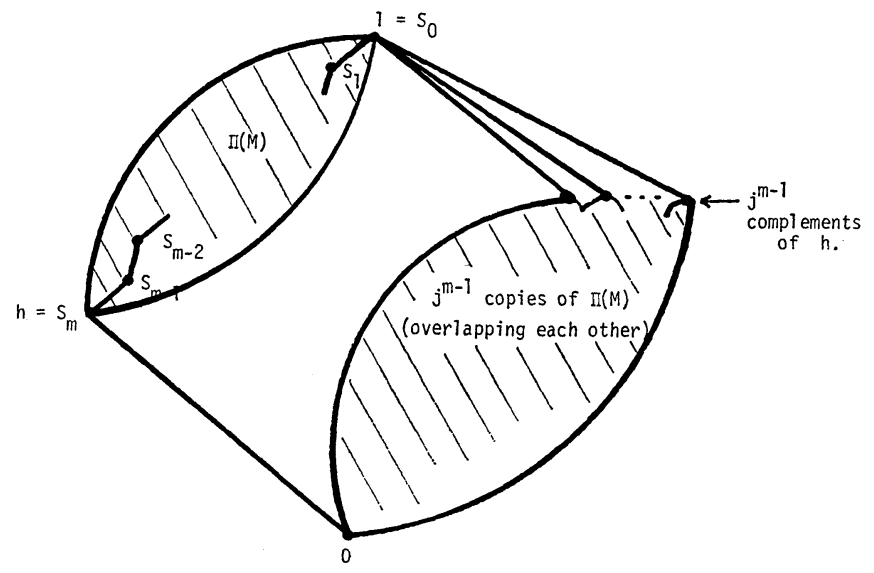

FiguRE 5

Let $\breve{G}$ denote the lattice of flats of the pregeometry $\left(\mathscr{B},{ }^{-}\right)$. We know that $\breve{G}$ is a geometric lattice. Define $\varphi: \widetilde{G} \rightarrow G$ as follow;

(1) $\varphi(\phi)=0$

(2) $\varphi(B(\beta ; f))=V_{G} B(\beta ; f)$

(3) $\varphi(A \cup B(\beta))=h \bigvee_{G}\left(V_{G} B(\beta)\right)=\sigma(\beta)$.

THEOREM 3. $\varphi$ is a lattice isomorphism and so $G$ is a geometric lattice. Some elemetary properties of the matroid given by $G$ are listed below:

A. Bases: If $I$ is a basis containing $h$ then $I-\{h\} \leqq B(f)$ for a unique function $f$. The set of $\rho(x)$ for $x \in I-\{h\}$ constitute $a$ basis for $\Pi(M)$.

If $I$ is a basis not containing $h$ then $I$ contains an element $y$ (not necessary unique) such that the set of $\rho(x)$ for $x \in I-\{y\}$ constitute a basis for $\Pi(M)$ and such that $V_{G}(I-\{y\})=c(f)$ for some function $f$.

B. Circuits: If $C$ is a circuit containing $h$ then the set of $\rho(x)$ such that $x \in C-\{h\}$ constitute a circuit in $\Pi(M)$. There is no function $f$ such that $x \leqq c(f)$ for all $x \in C-\{h\}$.

If $C$ is a circuit not containing $h$ then the set of $\rho(x)$ such that $x \in C$ constitute a circuit in $\Pi(M)$. There is a function $f$ such that $x \leqq c(f)$ for all $x \in C$.

C. Rank function: Let $\lambda_{G}$ denote the rank function of $G$ and let $\lambda$ denote the rank function of $\Pi(M)$. Let $S$ be a subset of $B \cup\{h\}$; write $S=S_{A} \cup S_{B}$ where $S_{B} \subseteq B$ and $S_{A}=\varnothing$ or $\{h\}$. Let 
Then

$$
\beta=\bigvee_{x \in S_{B}} \rho(x)
$$

$$
\lambda_{G}(S)= \begin{cases}0 & \text { if } S=\varnothing \\ \lambda(\beta) & \text { if } \quad S_{A}=\varnothing \text { and } \\ & S_{B} \subseteq B(f) \text { for some } f \in F\left(S_{B} \neq \varnothing\right) \\ 1+\lambda(\beta) & \text { otherwise. }\end{cases}
$$

Proof. It is easy to verify that $\phi$ is one-to-one, and onto. $\varphi$ is obviously order-preserving hence $\varphi$ is a lattice isomorphism. The matroid properties given in $\mathrm{A}, \mathrm{B}$ and $\mathrm{C}$ are clear; proofs are left to the reader.

CoRollary 1. Th is geometric iff $j$ is prime, or $m=1$.

Proof. If $j$ is prime then $\mathscr{C}=G$ and so the result follows from the last theorem. If $m=1$ then $\mathscr{C}$ is isomorphic to the Boolean algebra $B_{r}$ (i.e., lattice of divisors of $j$ ), and so $\mathscr{C l}$ is geometric.

Conversely, suppose $j$ is not prime and $m>1$. We show that is not geometric.

Consider the join of the two atoms $\alpha(1,2,1)$ and $\alpha(1,2,2)$. It is clear that these two do not both sit below $c(f)$ for some $f$ hence

$$
\alpha(1,2,1) V_{\mathscr{M}} \alpha(1,2,2)=\sigma(\beta)>h
$$

where $\beta=\{1,2\} /\{3\} / \cdots /\{m\}$. But since $j$ is not prime and $[0, h] \cong B_{r}$ we see that the rank of $h$ is at least 2 so the $\operatorname{rank}$ of $\sigma(\beta)$ is at least 3. So $\mathscr{C l}$ is not geometric.

Return to Figure 3 , where $j=2$ and $m=3$. Corollary 1 tells us that $\mathscr{C}$ is geometric in this case. In fact, its easy to check that this particular $\mathscr{K}$ is the projective plane of order 2.

5. The Birkhoff polynomial of $\mathscr{L}$. The purpose of this section is to determine the Birkhoff polynomial of $\mathscr{C}$. Some results in this section will be proved in a more general framework and then specialized to $\mathscr{M}$. We begin with some well-known facts about closure operators on lattices.

Let $K$ be a finite lattice with join and meet operations $V_{K}$ and $\Lambda_{K}$. Let $x \rightarrow \bar{x}$ be a closure operator and let $\bar{K}$ denote the set of closed elements of $K$. Then $\bar{K}$ is a lattice with join $\bigvee_{\bar{K}}$ and meet $\Lambda_{\bar{K}}$ given by

$$
\begin{aligned}
& x \bigvee_{\bar{K}} y=\overline{x \bigvee_{K} y} \\
& x \bigwedge_{K} y=x \bigwedge_{K} y .
\end{aligned}
$$


Let $h \in K$. Define $G(h)$ to be the set of elements of $K$ whose meet with $h$ is either 0 or $h$. Define a map $x \rightarrow \bar{x}$ from $K$ to $K$ by

$$
\bar{x}=\left\{\begin{array}{lll}
x & \text { if } & x \in G(h) \\
x \vee h & \text { if } & x \notin G(h) .
\end{array}\right.
$$

It is clear that $\bar{x} \geqq x$. Also ${ }^{-}$maps $K$ onto $G(h)$ so $\overline{\bar{x}}=\bar{x}$, and it is easy to check that if $x \geqq y$ then $\bar{x} \geqq \bar{y}$. Thus - is a closure on $K$ and the lattice of closed elements is $G(h)$. We sometimes write $G(h)=G_{0} \cup G_{h}$ where

$$
\begin{aligned}
& G_{0}=\{x \in K: x \wedge h=0\} \\
& G_{h}=\{x \in K: x \wedge h=h\} .
\end{aligned}
$$

Lemma 14. Suppose that $K$ is supersolvable with $M$-chain $C$, suppose $h \in C$ and let $C^{\prime}=C \cap G(h)$. Then $G(h)$ is supersolvable with $M$-chain $C^{\prime}$.

Proof. Let $\mathscr{D}$ be a chain in $G(h)$, and let $T$ be the sublattice of $G(h)$ generated by $\mathscr{D}$ and $C$. Note that $T$ is contained in the sublattice of $K$ generated by $C$ and $\mathscr{D}$ since $h \in C$. Also observe that $T$ is closed under joins in $K$, if $x, y \in T$ with $x \wedge h=y \wedge h=0$ then

$$
(x \underset{K}{\bigvee} y) \wedge h=(x \wedge h) \underset{K}{\bigvee}(y \wedge h)=0 \bigvee 0=0
$$

The first equality follows by the fact that $C$ is an $M$-chain for $K$.

Let $a, b$ and $c \in T$. Then

and

$$
\begin{aligned}
(a \underset{G}{\bigvee} b) \wedge c & =\left(a \bigvee_{K} b\right) \wedge c=(a \wedge c) \bigvee_{K}(b \wedge c) \\
& =(a \wedge c) \bigvee_{G}(b \wedge c)
\end{aligned}
$$

$$
\begin{aligned}
\left((a \wedge b) \bigvee_{G} c\right) & =(a \wedge b) \bigvee_{K} c=\left(a \bigvee_{K} c\right) \wedge\left(b \bigvee_{K} c\right) \\
& =\left(a \bigvee_{G} c\right) \wedge\left(b \bigvee_{G} c\right) .
\end{aligned}
$$

This proves the lemma.

Apply the last result to $\mathscr{C}$ with $h$ as in $\S \S 3$ and 4 . Note that $G=G(h)$ and so we see that $G$ is a supersolvable geometric lattice with $M$-chain

$$
0<h=s_{m-1}<s_{m-2}<\cdots<s_{1}<s_{0}=1 .
$$

We now use methods of Stanley to evaluate the Birkhoff polynomial of $\mathscr{l l}$.

Theorem 4. Let $B_{\mathscr{M}}(\lambda)$ denote the Birkhoff polynomial of $\mathscr{A}$. Then 


$$
B_{\mathscr{M}}(\lambda)=(\lambda-1)^{r}(\lambda-j)(\lambda-2 j) \cdots(\lambda-(m-1) j) .
$$

In particular $\mu_{m}(0,1)=\mu(j)\left((-1)^{m-1}(m-1) !\right) j^{m-1}$ where $\mu(j)$ denotes the number theoretic Möbius function.

Proof. Let $B_{h}(\lambda)$ denote the Birkhoff polynomial of the interval $[0, h]$. We first observe that

$$
B_{M}(\lambda)=B_{h}(\lambda)\left(\sum_{b \in G_{0}} \mu(0, b) \lambda^{m-r(b)}\right)
$$

where $r(b)$ denotes the rank of $b$. The proof is exactly the same as the proof of Theorem 2 given in Stanley [3]. In this proof Stanley assumes that the lattice $L$ under consideration is geometric whereas $\mathscr{C}$ is not in general geometric. However he only uses that $L$ is geometic to prove his Lemmas 1 and 2. Lemma 1 still holds since we've shown $h$ is modular in $\mathscr{t}$ (see Lemma 10). We now prove his Lemma 2; i.e., we show that for any $y \in \mathscr{l}, h \wedge y$ is a modular element of $[0, y]$.

Suppose $a \in[0, y]$ and $b \leqq a$. Then

$$
\begin{aligned}
(b \vee(y \wedge h)) \wedge a & =((b \vee h) \wedge y) \wedge a \text { by modularity of } h \\
& =((b \vee h) \wedge a)=b \vee(h \wedge a) \\
& =b \vee(h \wedge(y \wedge a))=b \vee((h \wedge y) \wedge a) .
\end{aligned}
$$

This part of the proof comes directly from Stanley [3, pg. 216]. Next suppose $b \leqq h \wedge y$ and $a \in[0, y]$. Then

$$
\begin{aligned}
b \vee((h \wedge y) \wedge a) & =b \vee(h \wedge a) \\
& =h \wedge(b \vee a) \\
& =h \wedge(y \wedge(b \vee a)) \text { since } b \vee a \leqq y \\
& =(h \wedge y) \wedge(b \vee a) .
\end{aligned}
$$

My thanks to Prof. R. P. Dilworth for suggesting this half of the proof.

This shows that

$$
B_{\mathscr{M}}(\lambda)=B_{h}(\lambda)\left(\sum_{b \in G_{0}} \mu(0, b) \lambda^{m-r(b)}\right) .
$$

Next consider the supersolvable geometric lattice $G$. As $h$ is a modular element of $G$ we can apply the same result again to $G$. This time the interval $[0, h]$ is isomorphic to a chain of length 1 so we have

$$
B_{G}(\lambda)=(\lambda-1)\left(\sum_{b \in G_{0}} \mu(0, b) \lambda^{m-r(b)}\right) .
$$

Combining this with the previous equation yields

$$
B_{M}(\lambda)=(\lambda-1)^{-1} B_{h}(\lambda) B_{G}(\lambda) .
$$


Also the interval $[0, h]$ in $M$ is isomorphic to the Boolean algebra $B_{r}$ so $B_{h}(\lambda)=(\lambda-1)^{r}$. Thus we have

$$
B_{M}(\lambda)=(\lambda-1)^{r-1} B_{G}(\lambda) .
$$

Recall that an $M$-chain for $G$ is $0<s_{m}<s_{m-1}<\cdots<s_{0}=1$. For $i=0$ to $m-1$, let $a_{i}$ denote the number of atoms of $G$ which are less than or equal to $s_{i}$ but not less than or equal to $s_{i+1}$. By Theorem 4.1 of Stanley [4, pg. 209] we know

$$
\begin{aligned}
B_{G}(\lambda) & =\left(\lambda-a_{m-1}\right)\left(\lambda-a_{m-2}\right) \cdots\left(\lambda-a_{0}\right) \\
& =(\lambda-1)\left(\lambda-a_{m-2}\right) \cdots\left(\lambda-a_{0}\right) .
\end{aligned}
$$

We next show that $a_{m-i}=(i-1) j$ for $i=2, \cdots, m$. The atoms of $G$ are $h$ together with all type $\mathrm{b}$ atoms $\mathscr{M}$. A type b atom $a$ is less than or equal to $s_{m-i}$ iff $\rho(a)<\rho\left(s_{m-i}\right)$. Now $\rho\left(s_{m-i}\right)$ has one block of size $i$ together with $m-i$ blocks of size 1 ; the block of size $i$ consists of $\{m, m-1, \cdots, m-i+1\}$.

Let $\alpha(p, q, r)$ be a type b atom with $\alpha(p, q, r) \leqq s_{m-i}$ and $\alpha(p, q, r) \rightrightarrows s_{m-i-1}$. Since $\alpha(p, q, r) \leqq s_{m-i}$ we know $p, q \in\{m, m-1, \cdots$, $m-i+1\}$. Since $\alpha(p, q, r) \leq s_{m-i-1}$ we know that $p$ and $q$ are not both members of $\{m, m-1, \cdots, m-i+2\}$. As $p<q$ we see

$$
\begin{aligned}
& p=m-i+1 \\
& q \in\{m, m-1, \cdots, m-i+2\} .
\end{aligned}
$$

Furthermore any choice of $q \in\{m, m-1, \cdots, m-i+2\}$ and $r \in$ $\{1,2, \cdots, j\}$ give a type b atom $\alpha(m-i+1, q, r)=a$ with $a \leqq s_{m-i}$ and $a \geqq s_{m-i-1}$. So $a_{m-i}=j(i-1)$. Thus

$$
B_{G}(\lambda)=(\lambda-1)(\lambda-j)(\lambda-2 j) \cdots(\lambda-(m-1) j)
$$

which together with equation (5.1) completes the proof of Theorem 4 .

Return now to Figure 3. Here $j=2$ and $m=3$ so we have

$$
B_{M}(\lambda)=(\lambda-1)(\lambda-2)(\lambda-4)=\lambda^{3}-7 \lambda^{2}+15 \lambda-8 .
$$

The interested reader can verify from Figure 3 that this is the correct Birkhoff polynomial for $\mathscr{K}$.

In Theorem 4 we obtained, for a nongeometric supersolvable lattice, factorization results similar to those which Stanley obtained for supersolvable geometric lattices. We can restate Theorem 4 in the following more general form.

THEOREM 4A. Let $(K, C)$ be a supersolvale lattice and let $h$ be an element of $C$. Suppose that $G(h)$ is a geometric lattice and that for each $y \in G_{0}$ the map from $[0, h]$ to $[y, y \vee h]$ given by $z \rightarrow z \vee y$ is one-to-one. Let $C^{\prime}=C \cap G(h)$ be 


$$
0<h=s_{0}<s_{1}<\cdots<s_{n}=1
$$

Then

$$
B(\lambda)=B_{h}(\lambda)\left(\lambda-a_{1}\right)\left(\lambda-a_{2}\right) \cdots\left(\lambda-a_{n}\right)
$$

where $a_{i}$ is the number of atoms a of $\mathscr{C l}$ which satisfy $a \leqq s_{i}, a \leqq s_{i-1}$.

The assumption that the map $z \rightarrow z \vee y$ is one-to-one is necessary. Consider for example

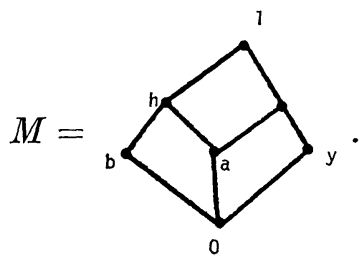

It is easy to check that $0<a<h<1$ is an $M$-chain for this lattice; note that the map from $[0, h]$ to $[y, y \vee h]$ given by $z \rightarrow z \vee h$ is not one-to-one ( $h$ and $b$ have the same image).

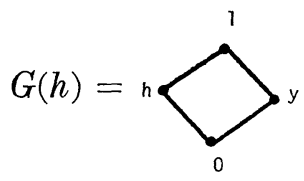

so $G(h)$ is geometric. It is easy to check that $a_{1}=1$ and $B_{h}(\lambda)=$ $(\lambda-1)^{2}$ so

$$
B_{h}(\lambda)\left(\lambda-a_{1}\right)=(\lambda-1)^{3} .
$$

However one can check that $B_{M}(\lambda)=\lambda(\lambda-1)(\lambda-2)$ and so Theorem $4 \mathrm{~A}$ does not hold.

\section{REFERENCES}

1. H. H. Crapo and G. C. Rota, On the Foundations of Combinatorial Theory: Combinatorial Geometries, M.I.T. Press, 1970.

2. P. Crawley and R. P. Dilworth, Algebraic Theory of Lattices, Prentice-Hall, 1973.

3. R. P. Stanley, Modular elements of geometric lattices, Algebra Universalis, VI (1971), 214-217.

4. — Supersolvable lattices, Algebra Universalis, II (1972), 197-217.

Received July 16, 1980.

California Institute of Technology

Pasadena, CA 91125 



\section{PACIFIC JOURNAL OF MATHEMATICS}

\section{EDITORS}

DoNALD BABBITT (Managing Editor)

University of California

Los Angeles, CA 90024

Hugo Rossi

University of Utah

Salt Lake City, UT 84112

C. C. MOORE and ANDREw OGG

University of California

Berkeley, CA 94720
J. DugundJI

Department of Mathematics

University of Southern California

Los Angeles, CA 90007

R. FinN and J. Milgram

Stanford University

Stanford, CA 94305

ASSOCIATE EDITORS
R. ARENS
E. F. BECKENBACH
B. H. NeumanN
F. WOLF
K. YoshidA

\section{SUPPORTING INSTITUTIONS}

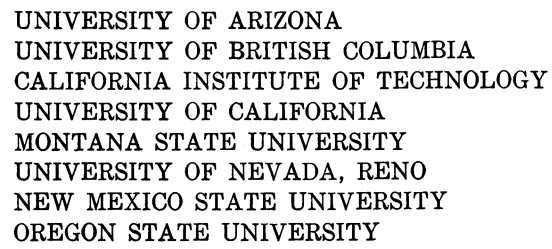

UNIVERSITY OF ARIZONA

UNIVERSITY OF BRITISH COLUMBIA

CALIFORNIA INSTITUTE OF TECHNOLOGY

UNIVERSITY OF CALIFORNIA

MONTANA STATE UNIVERSITY

UNIVERSITY OF NEVADA, RENO

NEW MEXICO STATE UNIVERSITY OREGON STATE UNIVERSITY

\author{
UNIVERSITY OF OREGON \\ UNIVERSITY OF SOUTHERN CALIFORNIA \\ STANFORD UNIVERSITY \\ UNIVERSITY OF HAWAII \\ UNIVERSITY OF TOKYO \\ UNIVERSITY OF UTAH \\ WASHINGTON STATE UNIVERSITY \\ UNIVERSITY OF WASHINGTON
}

The Supporting Institutions listed above contribute to the cost of publication of this Journal, but they are not owners or publishers and have no responsibility for its content or policies.

Mathematical papers intended for publication in the Pacific Journal of Mathematics should be in typed form or offset-reproduced, (not dittoed), double spaced with large margins. Please do not use built up fractions in the text of the manuscript. However, you may use them in the displayed equations. Underline Greek letters in red, German in green, and script in blue. The first paragraph or two must be capable of being used separately as a synopsis of the entire paper. Please propose a heading for the odd numbered pages of less than 35 characters. Manuscripts, in triplicate, may be sent to any one of the editors. Please classify according to the scheme of Math. Reviews, Index to Vol. 39. Supply name and address of author to whom proofs should be sent. All other communications should be addressed to the managing editor, or Elaine Barth, University of California, Los Angeles, California, 90024.

50 reprints to each author are provided free for each article, only if page charges have been substantially paid. Additional copies may be obtained at cost in multiples of 50 .

The Pacific Journal of Mathematics is issued monthly as of January 1966. Regular subscription rate: $\$ 102.00$ a year (6 Vols., 12 issues). Special rate: $\$ 51.00$ a year to individual members of supporting institutions.

Subscriptions, orders for numbers issued in the last three calendar years, and changes of address shoud be sent to Pacific Journal of Mathematics, P.O. Box 969, Carmel Valley, CA 93924, U.S.A. Old back numbers obtainable from Kraus Periodicals Co., Route 100, Millwood, NY 10546.

\footnotetext{
PUBLISHED BY PACIFIC JOURNAL OF MATHEMATICS, A NON-PROFIT CORPORATION

Printed at Kokusai Bunken Insatsusha (International Academic Printing Co., Ltd.). 8-8, 3-chome, Takadanobaba, Shinjuku-ku, Tokyo 160, Japan.
} 


\section{Pacific Journal of Mathematics}

\section{Vol. 96, No. 2 December, 1981}

Gerald A. Beer, A natural topology for upper semicontinuous functions and

a Baire category dual for convergence in measure $\ldots \ldots \ldots \ldots \ldots \ldots 251$

Georgia Benkart and J. Marshall Osborn, An investigation of real

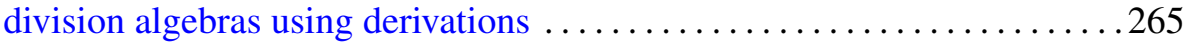

Donald Ian Cartwright and John R. McMullen, A structural criterion for the existence of infinite Sidon sets ........................ 301

Philip Hanlon, The fixed-point partition lattices $\ldots \ldots \ldots \ldots \ldots \ldots \ldots \ldots$

Eric Hayashi, The spectral density of a strongly mixing stationary Gaussian process

Chung-Wu Ho and Charles E. Morris, Jr., A graph-theoretic proof of

Sharkovsky's theorem on the periodic points of continuous functions . ...361

Sara Hurvitz, The automorphism groups of spaces and fibrations ....... 371

Atsushi Inoue, Schoichi Ota and Jun Tomiyama, Derivations of operator algebras into spaces of unbounded operators . .................. 389

Wolfgang B. Jurkat and Gary Sampson, On weak restricted estimates and endpoint problems for convolutions with oscillating kernels. I ........ 405

Georgios Koumoullis, Some topological properties of spaces of

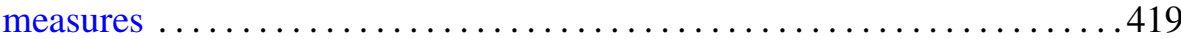

Wen Hsiung Lin, Algebraic Kahn-Priddy theorem ................. 435

Michael John McAsey, Invariant subspaces of nonselfadjoint crossed

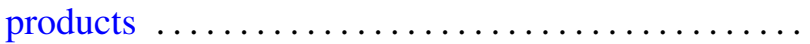

Justin Peters, Entropy of automorphisms on L.C.A. groups

Saburou Saitoh, A characterization of the adjoint $L$-kernel of Szegó type 